BMJ Open

Diabetes

Research

\& Care

\title{
One-hour plasma glucose combined with skin autofluorescence identifies subjects with pre-diabetes: the DIAPASON study
}

Lucia La Sala (1) , Elena Tagliabue, Paola de Candia, Francesco Prattichizzo, Antonio Ceriello

To cite: La Sala L, Tagliabue E, de Candia P, et al. One-hour plasma glucose combined with skin autofluorescence identifies subjects with prediabetes: the DIAPASON study. BMJ Open Diab Res Care 2020;8:e001331. doi:10.1136/ bmjdrc-2020-001331

- Additional material is published online only. To view, please visit the journal online (http://dx.doi.org/10.1136/ bmjdrc-2020-001331).

Received 12 March 2020 Revised 19 July 2020 Accepted 25 July 2020

\section{ABSTRACT}

Introduction The major challenge for diabetes prevention is early identification of individuals at risk to allow for implementation of measures to delay the onset of future disease. Measures such as fasting plasma glucose (FPG), 2-hour plasma glucose (2hPG), and glycosylated hemoglobin $(\mathrm{HbA} 1 \mathrm{c})$ are equally appropriate for identifying pre-diabetes and diabetes, but do not all identify the disease in the same individual. We tested the utility of a diagnostic method combining FPG, $2 \mathrm{hPG}$ and $\mathrm{HbA} 1 \mathrm{c}$ for early evaluation and easy identification of pre-diabetes. Research design and methods 531 subjects underwent skin autofluorescence (SAF) and glycemia analyses. We created two classification groups based on the American Diabetes Association diagnosis guidelines: (1) based on $2 \mathrm{hPG}$ and (2) based on a new combination of three glycemia parameters (the three-criteria strategy (3-c)). Logistic regression modeling was used to estimate the associations.

Results SAF showed high associations for both 3-c definition and $2 \mathrm{hPG}$ definition alone. These associations appeared stronger in 3-c than those in $2 \mathrm{hPG}$. The noninvasive SAF measurement outperformed $2 \mathrm{hPG}$ in the detection of dysglycemia or pre-diabetes. Stepwise selections identified 1-hour postload glucose (1hPG) as variable identifying pre-diabetes using the $2 \mathrm{hPG}$ criterion, and the model based on $1 \mathrm{hPG}$ plus SAF appeared to be the best association using the 3-c strategy.

Conclusions $1 \mathrm{hPG}$ coupled with SAF showed a strong association in the evaluation of pre-diabetes using the 3-c method.

\section{INTRODUCTION}

(C) Author(s) (or their employer(s)) 2020. Re-use permitted under CC BY-NC. No commercial re-use. See rights and permissions. Published by BMJ.

Department of Crdiovascular and Metabolic Disease, IRCCS MultiMedica, Milan, Italy

Correspondence to Dr Lucia La Sala; lucia.lasala@multimedica.it
Diabetes mellitus is considered a multifactorial, chronic metabolic disorder characterized by hyperglycemia owing to insulin resistance (IR) and insulin deficiency. ${ }^{1}$ Recent epidemiological data showed that diabetes affects people worldwide, reaching a prevalence of 412 million in 2015, and adding a plethora of people having risk factors that include impaired fasting glucose (IFG), impaired glucose tolerance (IGT), gestational diabetes and euglycemic IR this figure is expected to

\section{Significance of this study}

What is already known about this subject?

- Previous studies identified 1-hour postload glucose (1hPG) as a valuable risk factor for the development of pre-diabetes and type 2 diabetes mellitus, as well as cardiovascular disease.

What are the new findings?

- The prevalence of pre-diabetes is expected to increase worldwide.

- This cross-sectional study identifies the associations of $1 \mathrm{hPG}$ plus skin autofluorescence with dysglycemia and pre-diabetes.

- The novel method could be used to screen prediabetes in the general population.

How might these results change the focus of research or clinical practice?

- These results could represent a useful strategy to implement diabetes prevention.

increase. $^{2}$ From this view, it has been estimated that a substantial number of people, $50 \%$ of people in the general population 20-79 years of age globally, are unaware of their disease (http://www.diabetesatlas. org; International Diabetes Federation, IDF Diabetes Atlas Eighth Edition), exposing them to the risk of increased morbidity and mortality attributable to the onset of microvascular and macrovascular complications. Dysglycemia-also called glucose abnormalities (GAs) — which includes isolated IFG and/ or isolated IGT, represents a risk factor for developing diabetes and its related cardiovascular complications, which appear silently and several years before the clinical manifestation of the disease. ${ }^{3}$ Thus, promoting prevention strategies through early identification of subjects who are mostly free of clinical signs of diabetes should be considered a great public health priority to reduce the 
risk of diabetes and its associated burden. Recent clinical trials widely attested that lifestyle intervention ${ }^{3} 4$ or pharmacological therapy ${ }^{56}$ in subjects with IGT can prevent diabetes, providing a rationale for screening. Pre-diabetes, defined as IGT based on a 2-hour plasma glucose (2hPG) value of $140-199 \mathrm{mg} / \mathrm{dL}$ after a $75 \mathrm{~g}$ oral glucose tolerance test (OGTT), or IFG based on a fasting value of $100-125 \mathrm{mg} / \mathrm{dL}$ or glycosylated hemoglobin (HbAlc) between $5.7 \%$ and $6.4 \%(39-46 \mathrm{mmol} / \mathrm{mol}),{ }^{78}$ is a strong predictor of onset of cardiovascular and renal diseases. ${ }^{9}$ The consensus on the diagnostic definition of pre-diabetes is still debated ${ }^{10}$; thus, early identification of individuals at high risk of developing diabetes remains an open challenge. In recent years, 1-hour postload glucose (1hPG) has been recognized as a potent predictor of pre-diabetes, ${ }^{11-13}$ more than HbA1c, $2 \mathrm{hPG}$ and fasting plasma glucose (FPG) ${ }^{14-17}$ Moreover, few data are available on the possible role of skin autofluorescence (SAF) in detecting dysglycemia. ${ }^{18} \mathrm{SAF}$, a non-invasive measure of advanced glycated end-products (AGEs) associated with microvascular and macrovascular complications, ${ }^{19-22}$ has been suggested for opportunistic screening and early detection of pre-diabetes. ${ }^{23}$

In this work we sought to explore among the cohort of the Diabetes Prediction and Screening Observational (DIAPASON) study, at baseline, the associations between $1 \mathrm{hPG}$ and SAF and the prevalence of pre-diabetes using a strict method that combined 2hPG, FPG and HbA1c glycemic parameters for diagnosis.

\section{RESEARCH DESIGN AND METHODS}

Participants

The DIAPASON study is a clinical study about diabetes prevention, the primary endpoint of which was to estimate GA, diabetes and pre-diabetes frequencies by a procedure primarily based on evaluation of the diagnostic accuracy of SAF and HbA1c. A total of 1506 participants were selected on the basis of eligibility criteria by general practitioners in Milan; all subjects who filled in the Finnish Diabetes Risk Score (FINDRISC) questionnaire ${ }^{24}$ were invited for signed informed consent prior to laboratory screening. The eligibility criteria were as follows: age $40-75$ years and FINDRISC $\geq 9$ based on the results of the IGLOO study (to identify individuals with GAs).$^{25}$ The exclusion criteria were pre-existing diagnosis of diabetes and of any illness and/or medication, such as antidiabetes drugs, with a potential effect on the endpoints of the study. Additional exclusion criteria were adopted for SAF, such as skin changes, tattoos, excessive suntan, and use of bronzes or other sunless tanning products.

\section{Procedure}

The laboratory screening was attended by 531 participants at baseline, and body mass index (BMI), stature, blood pressure, OGTT at 60 and $120 \mathrm{~min}$ (1hPG and 2hPG, respectively), FPG, HbA1c, basal insulin, homeostasis model assessment for insulin resistance (HOMA-IR), lipid profile (total cholesterol, high-density lipoprotein, low-density lipoprotein, triacylglycerol), and microalbuminuria (MA) were assessed (online supplementary figure 1). SAF was measured as autofluorescence in human skin using an AGE Reader (DiagnOptics Technologies) to estimate the accumulation of AGEs in the skin. SAF was determined by the ratio between the light intensity reflected in the $420-600 \mathrm{~nm}$ wavelength range and the light intensity in the 300-420 wavelength range using the AGE Reader software. The cardiovascular risk score (CV risk) was calculated using the Progetto Cuore algorithm (www.cuore.iss.it). Participants were recruited between January 2013 and February 2017. All subjects gave written informed consent.

\section{Strategy for the definition of diagnostic groups}

We grouped subjects on the basis of two classification criteria that met the American Diabetes Association (ADA) guidelines: (1) the criteria based only on $2 \mathrm{hPG}$ definition; and (2) the criteria that we named the threecriteria strategy (3-c), which is a combined method based on FPG, 2hPG, and HbA1c. Specifically, FPG $<100 \mathrm{mg}$ / $\mathrm{dL}$ plus $2 \mathrm{hPG}<140 \mathrm{mg} / \mathrm{dL}$ plus $\mathrm{HbAlc}<5.7 \%$ identified subjects with normoglycemia (NGT); $100 \leq$ FPG $\leq 125$ $\mathrm{mg} / \mathrm{dL}$ plus $140 \leq 2 \mathrm{hPG} \leq 199 \mathrm{mg} / \mathrm{dL}$ plus $5.7 \leq \mathrm{HbAlc}$ $\leq 6.4 \%$ identified pre-diabetes (PRE); FPG $>125 \mathrm{mg} / \mathrm{dL}$ plus $2 \mathrm{hPG}>199 \mathrm{mg} / \mathrm{dL}$ plus HbAlc $>6.4 \%$ identified type 2 diabetes (T2D); and subjects not satisfying any of the three conditions were considered to be in the group of miscellaneous glycemic abnormalities (mGAs).

\section{Plasma separation and laboratory testing}

Approximately $5 \mathrm{~mL}$ of venous blood was extracted in an EDTA anticoagulant tube at room temperature. The venous blood sample was centrifuged at $3000 \times g$. FPG was detected by the Slein method using a Siemens analyzer (Germany). We used OGTT to assess the 2hPG and $1 \mathrm{hPG}$ values. Triacylglycerol and total cholesterol were measured using an automated enzymatic colorimetric test (Siemens). HbA1c was detected by a high-performance liquid chromatography automated system (Tosoh, Japan). Insulinemia levels were detected by a Centaur XP analyzer (Siemens). HOMA-IR was calculated by the formula 'FPG $(\mathrm{mg} / \mathrm{dL}) \times$ fasting insulin $(\mathrm{uU} / \mathrm{mL}) / 405$ '. MA was detected in urine samples previously centrifuged for $10 \mathrm{~min}$ at $3000 \times g$ to avoid cellular debris using an IMMAGE instrument (Beckman Coulter).

\section{Statistical analysis}

Continuous variables are presented as mean and SD, and their distributions were assessed for normality using the Kolmogorov-Smirnov test. All normally distributed variables were compared between groups using one-way analysis of variance and paired contrasts. Non-normally distributed variables were compared between groups using the Kruskal-Wallis test and pairwise two-sample Wilcoxon comparisons. Sex, as the only categorical 
Table 1 Prevalence of IFG, IGT and T2D newly diagnosed by ADA recommendations

\begin{tabular}{llcc}
\hline & NGT & IGT & T2D \\
\hline 2hPG & $397(75 \%)$ & $98(18 \%)$ & $34(6 \%)$ \\
FPG & $437(82 \%)$ & $88(17 \%)$ & $6(1 \%)$ \\
HbA1c & $150(28 \%)$ & $324(61 \%)$ & $57(11 \%)$ \\
\hline
\end{tabular}

$\mathrm{HbA1c}$ was able to detect IGT and newly diagnosed T2D with a major percentage than $2 \mathrm{hPG}$ and FPG. Conversely, $2 \mathrm{hPG}$ and FPG were able to identify more subjects with normoglycemia than HbA1c.

ADA, American Diabetes Association; FPG, fasting plasma glucose; HbA1c, glycosylated hemoglobin; 2hPG, 2-hour plasma glucose; IFG, impaired fasting glucose; IGT, impaired glucose tolerance; NGT, normoglycemia; T2D, type 2 diabetes.

variable, was compared between groups using the $\chi^{2}$ test or Fisher's exact test, as appropriate. Cohen's weighted kappa was used to test for agreement among FPG, 2hPG and HbA1c. The Spearman correlation matrix was calculated for all collected variables. For both classification criteria (diagnosis based on 2hPG or the 3-c), we calculated the 1hPG and SAF best cut-offs using Youden's index and tested the sensitivity, specificity, positive and negative predicted values, positive and negative likelihood ratio, and the area under the receiver operating characteristic (ROC) curve (AUC). Stepwise forward regression models were also performed, and ROC curves were drawn for the selected models. Finally, to evaluate the predictive power of $1 \mathrm{hPG}$ and SAF in discriminating between diagnostic groups (3-c), we performed multivariable logistic models, and ORs adjusted for age, sex and BMI were calculated. ROC curves for logistic models were drawn, and AUCs with 95\% CI were calculated. Statistical significance was defined as $\mathrm{p}<0.05$. Statistical analyses were carried out with SAS V.9.4 software.

\section{RESULTS}

Distribution of diagnostic groups based on different criteria

The prevalence of pre-diabetes based on FPG only did not differ notably from that based on 2hPG, whereas HbAlc $\%$ classified more cases of pre-diabetes $(61 \%)$ than the other diagnostic criteria (table 1), suggesting that HbA1c is able to classify a larger proportion of subjects as diabetics and pre-diabetics than 2hPG or FPG, while FPG identified more people with normoglycemia. 2hPG identified a higher prevalence of newly diagnosed cases of T2D $(6 \%)$ than FPG $(1 \%)$, whereas HbAlc reached $11 \%$ prevalence, which was higher than others.

To assess the consistency of the measuring process according to the same diagnostic result, we measured the agreements among FPG, 2hPG or HbAlc metrics using Cohen's weighted kappa coefficient $(\mathrm{k})$, as shown in table 2. The data showed slight/fair agreement $(k<0.4)$, suggesting that the agreement between the criteria was no better than that which would be obtained by chance alone, in all cases.
Table 2 Cohen's weighted $\mathrm{k}$ agreement among FPG, 2hPG and $\mathrm{HbA} 1 \mathrm{c}$

\begin{tabular}{lll}
\hline & Weighted kappa & Symmetry \\
\hline FPG vs 2hPG & $0.35(0.27-0.44)$ & $<0.0001$ \\
FPG vs HbA1c & $0.11(0.08-0.15)$ & $<0.0001$ \\
2hPG vs HbA1c & $0.17(0.12-0.23)$ & $<0.0001$ \\
\hline
\end{tabular}

FPG, fasting plasma glucose; $\mathrm{HbA} 1 \mathrm{c}$, glycosylated hemoglobin; 2hPG, 2-hour plasma glucose.

We noticed that SAF values were highly significant when detecting pre-diabetes using the FPG definition only, but in HbA1c only SAF values were significantly high not only in pre-diabetes classification but also in new T2D (online supplementary tables S1-S3). In online supplementary figure $1, \mathrm{SAF}$ revealed a significant correlation with all glycemic parameters, reaching the highest statistical level with $\mathrm{HbA1c}$ values $(\mathrm{p}<0.0001)$, providing rationale for the use of SAF in detecting glycemic exposures. Also, the correlation matrix exhibited a correlation with SAF and all glycemic parameters, in particular HbAlc $(p<0.001)$, triacylglycerol, HOMA-IR and MA (online supplementary figure 1), supporting the potential of SAF for monitoring the early development of diseases related to derangements in glucose homeostasis.

\section{Combined three-criteria (3-c) strategy}

To explore whether 3-c (FPG, 2hPG and HbAlc) identifies subjects with different pathogenic mechanisms, we divided the population into four groups (NGT, PRE, newly diagnosed and untreated T2D, and mGA) based on the ADA guidelines, as explained in the 'Research design and methods' section. Thus, we classified 126 subjects with NGT, 377 with mGA, 24 with PRE and 4 subjects with diabetes (who were excluded from major analyses due to the low representative number). Subjects fulfilling the three diagnostic criteria for pre-diabetes had a significantly higher HOMA index, worse insulin secretion, reduced high-density lipoprotein, increased BMI, increased $1 \mathrm{hPG}$, increased SAF, and increased CV risk compared with NGT (table 3). Subjects classified as NGT with 3-c had the most favorable cardiometabolic parameters.

\section{$1 \mathrm{hPG}$ and SAF identify dysglycemia in 3-c}

We performed logistic regression models to evaluate the association between $1 \mathrm{hPG}, \mathrm{SAF}$ and diagnostic groups (3-c) adjusted for age, sex and BMI. ROC curves and ORs are shown in figure $1 \mathrm{~A}, \mathrm{~B}$. $1 \mathrm{hPG}$ and SAF were significantly associated with an increased prevalence of mGA and PRE, if compared with NGT (mGA: 1hPG OR 1.02, $\mathrm{p}<0.0001$ and SAF OR 2.23, $\mathrm{p}<0.01$; PRE: $1 \mathrm{hPG}$ OR 1.07, $\mathrm{p}<0.0001$ and SAF OR 11.57, $\mathrm{p}<0.01)$. We also calculated the optimal cut-off value and determined the accuracy of $1 \mathrm{hPG}$, identifying values $\geq 157 \mathrm{mg} / \mathrm{dL}$ as discriminative between mGA and NGT (AUC=0.71, $<<0.0001$ ); for $\mathrm{SAF}$, the cut-off was $\geq 2.1$ intrinsic fluorescence units 


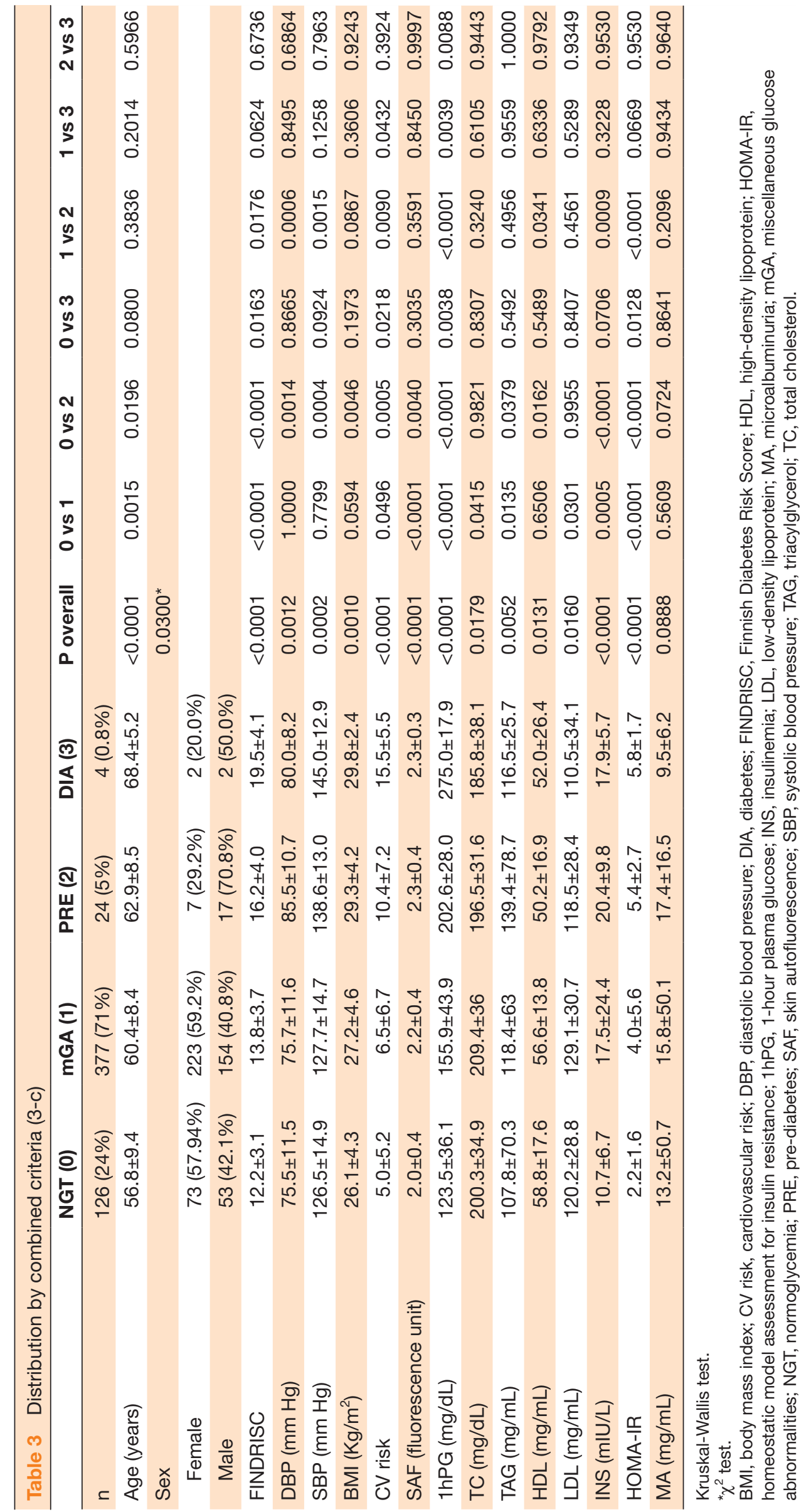


A

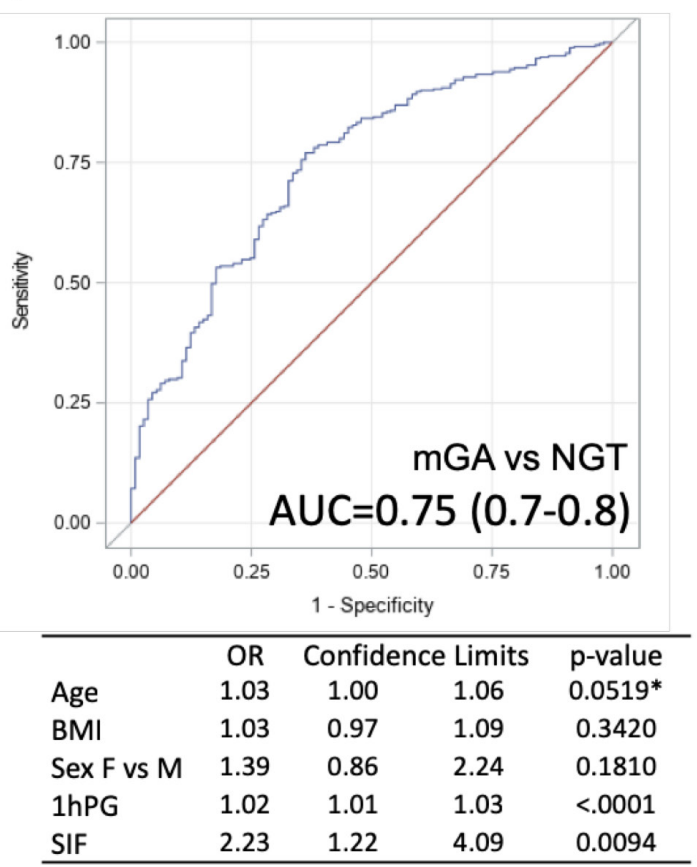

B

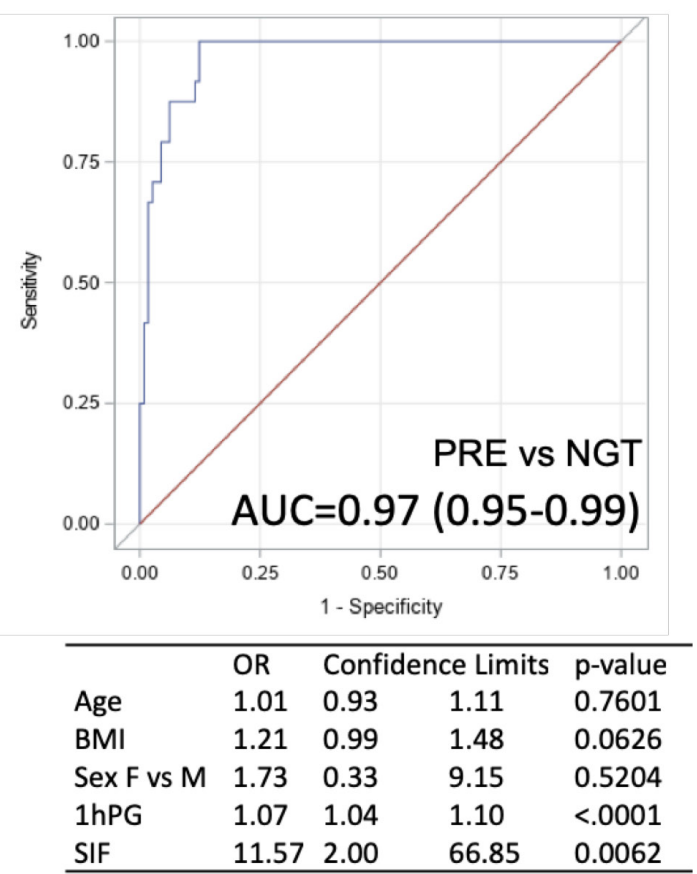

Figure 1 Multivariable logistic models for (A) miscellaneous glucose abnormalities versus normoglycemia and (B) impaired glucose tolerance (pre-diabetes) versus normoglycemia. * ${ }^{*}$-value borderline significant. AUC, area under the curve; BMI, body mass index; F, female; 1hPG, 1-hour postload glucose; M, male; mGA, miscellaneous glucose abnormalities; NGT, normoglycemia; PRE, pre-diabetes; SIF, skin intrinsic fluorescence.

for detecting mGA versus NGT (AUC=0.63, $\mathrm{p}=0.0002$ ) (table 4).

\section{$1 \mathrm{hPG}$ as common predictor for both 3-c and 2hPG criteria}

Using the 2hPG-only criteria, the stepwise forward regression model identified $1 \mathrm{hPG}$ as the best predictor (T2D vs IGT: OR 1.05 (1.03-1.07), $\mathrm{p}<0.0001$, AUC $=0.87$; T2D vs NGT: OR 1.09 (1.06-1.13), $\mathrm{p}<0.0001, \mathrm{AUC}=0.98)$. However, for IGT versus NGT, diastolic blood pressure (OR 1.06 (1.03-1.09), $\mathrm{p}=0.0002$ ) and 1hPG (OR 1.04 (1.03-1.05), $\mathrm{p}<0.0001)$ were identified as the two most influential variables, with a global AUC of 0.86 (online supplementary figure $3 \mathrm{~A}-\mathrm{C}$ ). We also calculated the optimal cut-off values and determined the accuracy of $1 \mathrm{hPG}$ in identifying diagnostic groups based on 2hPG criterion only. We identified a cut-off of $1 \mathrm{hPG} \geq 154 \mathrm{mg} /$ $\mathrm{dL}$ for detecting pre-diabetes versus NGT $(\mathrm{AUC}=0.84$, $\mathrm{p}<0.0001)$ and $1 \mathrm{hPG} \geq 224 \mathrm{mg} / \mathrm{dL}$ for detecting T2D versus pre-diabetes $(\mathrm{AUC}=0.86, \mathrm{p}<0.0001$; online supplementary table S4). After adjusting for age, sex and BMI, the cut-off values did not differ from those of the unadjusted model (data not shown).

\section{DISCUSSION}

In this work, we proposed a method (3-c) consisting of the concomitant use of the three canonical metrics useful for detecting hyperglycemia (FPG, 2hPG and HbAlc) on the identification of pre-diabetes in the DIAPASON study. Our data demonstrated that the 3-c method could outperform a single glycemic parameter used alone.
This method takes place from our observations about glycemic definitions (based on only one or two parameters), outlining a considerably different prevalence of individuals at risk of developing pre-diabetes or T2D (tables 1 and 2).

For the first time, we reported that when we adopted the 3-c method, the differences in the pre-diabetic phenotype were more evident than when single definitions were used alone. Using this novel approach, we identified a phenotype of individuals with normoglycemia having a more realistic normal cardiometabolic trait, characterized by lower levels of IR and triacylglycerol, compared with those identified using the other glycemic definitions (online supplementary tables S1-S3). Also, 1hPG and SAF measurements exhibited reduced levels in NGT as defined by 3-c compared with those in 2hPG only.

Additionally, we found a relevant prevalence of individuals in the intermediate status defined as mGA-between normoglycemic and pre-diabetic-having a different cardiometabolic trait than individuals with pre-diabetes and normoglycemia (table 3). Subjects with mGA would have been classified erroneously if they had been identified by $2 \mathrm{hPG}$ criterion, only leading to misclassification of some individuals, rather than by the strict classification performed with 3-c. We identified for the first time a cut-off threshold for SAF of $\geq 2.1$ (mGA vs NGT) (table 4 ) and a cut-off threshold for $1 \mathrm{hPG}$ of $\geq 157 \mathrm{mg} / \mathrm{dL}$ for discriminating mGA from NGT, which do not differ from that observed using $2 \mathrm{hPG}$ definition only (1hPG $\geq 154 \mathrm{mg} / \mathrm{dL}$ for pre-diabetes vs normoglycemia; online 


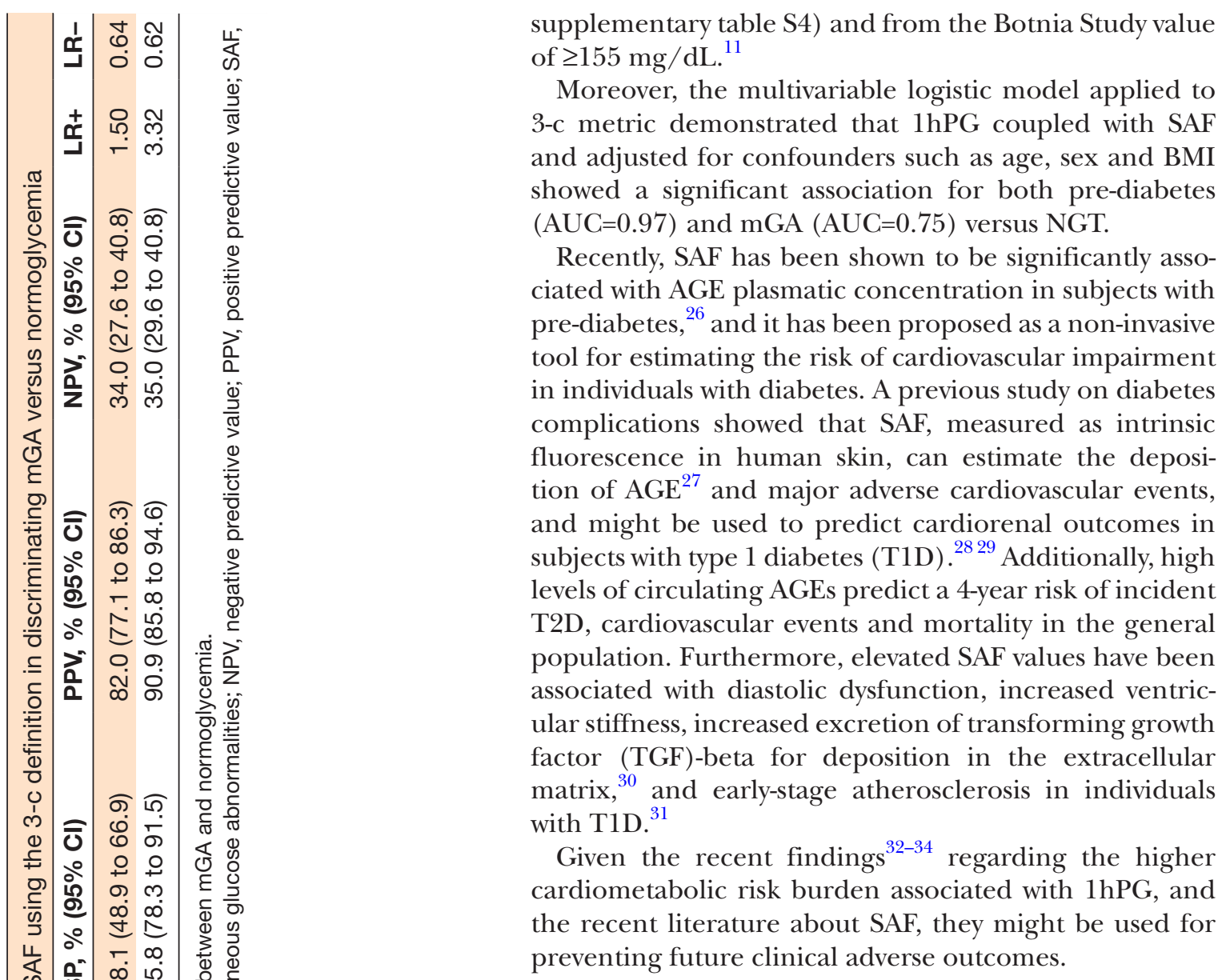

\section{Strengths and limitations}

Some strengths and limitations should be considered in the present study. A strength is that we conducted standardized biochemical measurement evaluations of blood samples which were analyzed in a certified laboratory. Further, all participants completed the examinations, and the percentage of missing values was relatively small. However, some limitations exist. In this observational cohort study, further analyses will be required to evaluate the risk of development of pre-diabetes or diabetes and its associated cardiovascular complications. Nevertheless, some risk factors for high SAF values were not taken into account, such as rheumatic disease and depression.

\section{CONCLUSION}

The model of SAF and 1hPG represented by this study acts as a good predictor of people at risk. Our results provide evidence for planning monitoring and prevention programs based on SAF, simply by scanning AGE level on the skin, and 1hPG, which is more suitable in clinical settings than 2hPG. Using $1 \mathrm{hPG}$ and SAF might predict and identify a high number of people at risk and might be the first step toward identifying general populations at risk using more extensive screening programs. Specifically, the association values of $1 \mathrm{hPG}$ and SAF might be effective in screening individuals in the early stage of pre-diabetes. 
Acknowledgements The authors wish to thank Alessandra Panvini-Rosati for database assistance.

Contributors LLS contributed to conception and design of the study, analysis and interpretation of data, and wrote the manuscript. LLS and ET performed statistical analyses. FP and PC critically revised the manuscript. AC contributed to conception and provided critical revision of the paper for important intellectual content. All authors read and approved the manuscript submission. LLS takes responsibility for the content of the article.

Funding This work has been supported by EFSD/Sanofi 2017 (to LLS), Fondazione 'Romeo ed Enrica Invernizzi' (Milan, Italy), Italian Ministry of Health 'Ricerca Corrente' to IRCCS MultiMedica and 'RF2016 - 02364513' (to AC).

Competing interests None declared.

Patient consent for publication Not required.

Ethics approval The DIAPASON protocol was approved by the institutional review boards/independent ethics committee of the IRCCS MultiMedica (protocol number 24/2012(153)).

Provenance and peer review Not commissioned; externally peer reviewed.

Data availability statement All data relevant to the study are included in the article or uploaded as supplementary information. Our data are not in a repository. We have permission from the participants in terms of informed consent.

Open access This is an open access article distributed in accordance with the Creative Commons Attribution Non Commercial (CC BY-NC 4.0) license, which permits others to distribute, remix, adapt, build upon this work non-commercially, and license their derivative works on different terms, provided the original work is properly cited, appropriate credit is given, any changes made indicated, and the use is non-commercial. See: http://creativecommons.org/licenses/by-nc/4.0/.

ORCID iD

Lucia La Sala http://orcid.org/0000-0002-7580-1377

\section{REFERENCES}

1 Wang P, Fiaschi-Taesch NM, Vasavada RC, et al. Diabetes mellitus-advances and challenges in human $\beta$-cell proliferation. Nat Rev Endocrinol 2015;11:201-12.

2 Bloomgarden Z. Questioning glucose measurements used in the International diabetes Federation (IDF) atlas. J Diabetes 2016;8:746-7.

3 Tuomilehto J, Lindström J, Eriksson JG, et al. Prevention of type 2 diabetes mellitus by changes in lifestyle among subjects with impaired glucose tolerance. N Engl J Med 2001;344:1343-50.

4 American Diabetes Association and National Institute of Diabetes, Digestive and Kidney Diseases. The prevention or delay of type 2 diabetes. Diabetes Care 2002;25:742-9.

5 Gillies CL, Abrams KR, Lambert PC, et al. Pharmacological and lifestyle interventions to prevent or delay type 2 diabetes in people with impaired glucose tolerance: systematic review and metaanalysis. BMJ 2007;334:299.

6 Knowler WC, Barrett-Connor E, Fowler SE, et al. Reduction in the incidence of type 2 diabetes with lifestyle intervention or metformin. N Engl J Med 2002;346:393-403.

7 American Diabetes Association. (2) classification and diagnosis of diabetes. Diabetes Care 2015;38 Suppl:S8-16.

8 American Diabetes Association. Diagnosis and classification of diabetes mellitus. Diabetes Care 2014;37 Suppl 1:S81-90.

9 Ali MK, Bullard KM, Saydah S, et al. Cardiovascular and renal burdens of prediabetes in the USA: analysis of data from serial cross-sectional surveys, 1988-2014. Lancet Diabetes Endocrinol 2018;6:392-403.

10 Bergman M, Manco M, Sesti G, et al. Petition to replace current OGTT criteria for diagnosing prediabetes with the 1-hour post-load plasma glucose $\geq 155 \mathrm{mg} / \mathrm{dl}(8.6 \mathrm{mmol} / \mathrm{L})$. Diabetes Res Clin Pract 2018;146:18-33.

11 Abdul-Ghani MA, Lyssenko V, Tuomi T, et al. Fasting versus postload plasma glucose concentration and the risk for future type 2 diabetes: results from the Botnia study. Diabetes Care 2009;32:281-6.

12 Fiorentino TV, Marini MA, Andreozzi F, et al. One-Hour Postload hyperglycemia is a stronger predictor of type 2 diabetes than impaired fasting glucose. J Clin Endocrinol Metab 2015;100:3744-51.
13 Abdul-Ghani MA, Williams K, DeFronzo RA, et al. What is the best predictor of future type 2 diabetes? Diabetes Care 2007;30:1544-8.

14 Jagannathan R, Sevick MA, Fink D, et al. The 1-hour postload glucose level is more effective than $\mathrm{HbA} 1 \mathrm{c}$ for screening dysglycemia. Acta Diabetol 2016;53:543-50.

15 Abdul-Ghani MA, DeFronzo RA. Plasma glucose concentration and prediction of future risk of type 2 diabetes. Diabetes Care 2009;32 Suppl 2:S194-8.

16 Dankner R, Abdul-Ghani MA, Gerber Y, et al. Predicting the 20-year diabetes incidence rate. Diabetes Metab Res Rev 2007;23:551-8.

17 Alyass A, Almgren P, Akerlund M, et al. Modelling of OGTT curve identifies $1 \mathrm{H}$ plasma glucose level as a strong predictor of incident type 2 diabetes: results from two prospective cohorts. Diabetologia 2015;58:87-97.

18 Tentolouris N, Lathouris $\mathrm{P}$, Lontou $\mathrm{S}$, et al. Screening for HbA1cdefined prediabetes and diabetes in an at-risk Greek population: performance comparison of random capillary glucose, the ADA diabetes risk test and skin fluorescence spectroscopy. Diabetes Res Clin Pract 2013;100:39-45.

19 Conway BN, Aroda VR, Maynard JD, et al. Skin intrinsic fluorescence is associated with coronary artery disease in individuals with long duration of type 1 diabetes. Diabetes Care 2012;35:2331-6.

20 Monnier VM, Bautista O, Kenny D, et al. Skin collagen glycation, glycoxidation, and crosslinking are lower in subjects with long-term intensive versus conventional therapy of type 1 diabetes: relevance of glycated collagen products versus $\mathrm{HbA} 1 \mathrm{c}$ as markers of diabetic complications. DCCT skin collagen ancillary Study Group. diabetes control and complications trial. Diabetes 1999;48:870-80.

21 Orchard TJ, Lyons TJ, Cleary PA, et al. The association of skin intrinsic fluorescence with type 1 diabetes complications in the DCCT/EDIC study. Diabetes Care 2013;36:3146-53.

22 Jacobs K, Navarrete Santos A, Simm A, et al. The skin autofluorescence reflects the posttranslational glycation grade of the matrix protein collagen. Free Radic Biol Med 2014;75 Suppl 1:S34.

23 Maynard JD, Rohrscheib M, Way JF, et al. Noninvasive type 2 diabetes screening: superior sensitivity to fasting plasma glucose and A1c. Diabetes Care 2007:30:1120-4.

24 Tuomilehto J, Lindström J, Hellmich M, et al. Development and validation of a risk-score model for subjects with impaired glucose tolerance for the assessment of the risk of type 2 diabetes mellitus-The STOP-NIDDM risk-score. Diabetes Res Clin Pract 2010;87:267-74.

25 Franciosi M, De Berardis G, Rossi MCE, et al. Use of the diabetes risk score for opportunistic screening of undiagnosed diabetes and impaired glucose tolerance: the IGLOO (impaired glucose tolerance and long-term outcomes observational) study. Diabetes Care 2005;28:1187-94.

26 Liu C-Y, Huang Q-F, Cheng Y-B, et al. A comparative study on skin and plasma advanced glycation end products and their associations with arterial stiffness. Pulse 2017:4:208-18.

27 van Waateringe RP, Fokkens BT, Slagter SN, et al. Skin autofluorescence predicts incident type 2 diabetes, cardiovascular disease and mortality in the general population. Diabetologia 2019;62:269-80.

28 Blanc-Bisson C, Velayoudom-Cephise FL, Cougnard-Gregoire A, et al. Skin autofluorescence predicts major adverse cardiovascular events in patients with type 1 diabetes: a 7-year follow-up study. Cardiovasc Diabetol 2018;17:82.

29 Vélayoudom-Céphise F-L, Rajaobelina K, Helmer C, et al. Skin autofluorescence predicts cardio-renal outcome in type 1 diabetes: a longitudinal study. Cardiovasc Diabetol 2016;15:127.

30 Striker LJ, Striker GE. Administration of ages in vivo induces extracellular matrix gene expression. Nephrol Dial Transplant 1996;11 Suppl 5:62-5.

31 Osawa S, Katakami N, Kuroda A, et al. Skin autofluorescence is associated with early-stage atherosclerosis in patients with type 1 diabetes. J Atheroscler Thromb 2017;24:312-26.

32 Fiorentino TV, Sesti F, Andreozzi F, et al. One-Hour post-load hyperglycemia combined with $\mathrm{HbA} 1 \mathrm{c}$ identifies pre-diabetic individuals with a higher cardio-metabolic risk burden. Atherosclerosis 2016;253:61-9.

33 Lowe LP, Liu K, Greenland P, et al. Diabetes, asymptomatic hyperglycemia, and 22-year mortality in black and white men. The Chicago heart association detection project in industry study. Diabetes Care 1997;20:163-9.

34 Succurro E, Marini MA, Arturi F, et al. Elevated one-hour post-load plasma glucose levels identifies subjects with normal glucose tolerance but early carotid atherosclerosis. Atherosclerosis 2009;207:245-9. 